\title{
Anomalous transfer effects following pattern discrimination training in the squirrel monkey
}

\begin{abstract}
P.C. DODWELL, J. S. LITNER, and R. R. NIEMI, Queen's University at Kingston, Kingston, Ont., Canada
\end{abstract}

Two squirrel monkeys (Samiri scuireus) were trained to discriminate between two patterns that were identical, except that their orientations differed by $90 \mathrm{deg}$. In subsequent transfer tests (a) the training-positive shape was shown with a novel shape having the same "positive" orientation, and (b) the training-negative shape was shown with the same novel shape in the "negative" orientation. Transfer choices were anomalous; the novel shape was preferred to the training-positive shape, and the training-negative shape was preferred to the novel "negative" shape. These results are held to be a function of the contour-coding processes of the mammalian visual system.

Normally, one expects an organism that has been trained to discriminate between two stimuli to choose the training-positive stimulus if it is shown in a subsequent transfer pair or to avoid the training-negative stimulus if it is shown. Relational leaming, or transpositional discrimination, may be an exception to this generalization under some conditions, but in these cases, the dimension along which the transposition occurs is usually unitary and easily identified. Krechevsky (1938) discovered an anomalous transfer situation, in which a novel shape was preferred to the training-positive shape but where the relevant stimulus dimension (if any) was not obvious. He trained rats to discriminate between square arrays of small squares arranged in "rows" or in "columns," as shown in Fig. 1, i.e., the horizontal center-to-center distance between squares is smaller than the vertical center-to-center distance in the "row" array, and vice versa for "columns." A group of rats trained with "rows" positive was subsequently presented with a choice between rows and an equally bright pattern of smooth horizontal stripes, and reliably preferred the stripes. Another group trained with "columns" positive subsequently preferred smooth vertical stripes to "columns." Dodwell (1965) replicated this finding (also with rats) and, moreover, showed that transfer preferences were anomalous with respect to the negative shape; rats trained with "rows" negative subsequently preferred "rows" to horizontal stripes and similarly for "columns" and vertical between trials, or pressing both simultaneously, was effectively suppressed by introducing a time-out of $2 \mathrm{~min}$ following such behavior. Otherwise, the intertrial interval was $30 \mathrm{sec}$, and food reinforcement was used. One $S$ developed a side preference that was shaped out before further training.

Following shaping, "initial preference" tests were run without reinforcement. One $S$ was presented with choice between "rows" and horizontal stripes of the same overall brightness, the other with choice between "columns" and vertical stripes, each for 40 trials. On the next day, the procedure was reversed so that the first $S$ was presented with a choice between "columns" and vertical stripes, the second $S$ with a choice between "rows" and horizontal stripes. There were no reliable initial preferences. During these preference sessions, and in all subsequent sessions, stimuli were randomized for side of presentation under the restriction that no stimulus was ever presented more than three times consecutively on the same side. Nonreinforcement of the preference trials led to sluggish responding, so both Ss were reshaped for 2 days before discrimination training began.

Discrimination training consisted of 40 presentations per day on the "rows" vs "columns" discrimination, the "rows" being positive (reinforced) for $S 1$ and the "columns" positive for $\mathbf{S 2}$. The only punishment was a 2-min time-out after an incorrect choice; otherwise, the intertrial interval was $30 \mathrm{sec}$, as in shaping, and reinforcement was given on $80 \%$ of correct trials (one in every five correct choices, chosen randomly, was not reinforced). S1 reached a very stringent criterion of $40 / 40$ correct on 2 consecutive days after 340 trials, $S 2$ after 520 trialf:

Transfer tests were administered on the 2 days after $S 2$ had reached criterion. On the 1st transfer day, the test was between "rows" and horizontal stripes, and on the 2nd day, it was between "columns" and vertical stripes; thus, $S 1$ had a transfer test on its positive shape first, followed the next day by the test on its negative shape, and vice versa for $S 2$. On both transfer days, each $S$ was given 100 trials, which consisted of the original discrimination training procedure, with the 20 transfer tests (without reinforcement) substituted for the previously unreinforced $20 \%$ of correct choices. This was done in order to disrupt the original discrimination as little as possible and to insure vigorous responding to the transfer shapes.

\section{RESULTS}

Fig. 1. (a) The training patterns, called "rows" and "columns." (b) A transfer pair; "columns" and vertical stripes.
S1 preferred horizontal stripes to "rows" 19/20 times $(p<.01$ on an exact binomial test, with $p=q=1 / 2$ ) and 
"columns" to vertical stripes $18 / 20$ times $(\mathrm{p}<.01)$, thus demonstrating very reliable anomalous transfer to both positive and negative training shapes. $S 2$, on the other hand, preferred horizontal stripes to "rows" (its negative shape) on 13/20 trials (nonsignificant) but preferred vertical stripes to "columns" $19 / 20$ times $(p<.01)$ on the following day. $S 2$ thus showed a very reliable positive anomalous effect but no negative effect, although responding to the negative shape was at a much higher level than on ordinary training trials. Both Ss maintained their discrimination between the training stimuli at nearly $100 \%$ correct on the transfer days.

It is surprising that one $S$ should show such strong negative anomalous transfer, and the other $S$ none. The order of presentation of transfer tests was balanced; S1 had transfer tests with the positive shape, followed by tests with the negative shape on the next day, but $S 2$ had tests on the negative shape first. To find out whether or not this was relevant to S2's failure to show negative anomalous transfer, both Ss were retrained to criterion after several days' rest, and the transfer tests were administered in reversed order for the two monkeys. This time, S1 failed to show negative anomalous transfer when the transfer test with the negative shape was given first, but S2 did show it, and again, both showed reliable positive aromalous transfer. To make quite certain that this order effect was real, the whole procedure was repeated again, and with the same result: Provided transfer on the positive shape preceded the negative, the latter showed the anomalous effect but not otherwise. It is as if the system responsible for the anomalies has to be "primed" on the positive transfer before it will respond fully on the negative.

\section{DISCUSSION}

How may the anomalous transfer effects be explained? They can be explained by a contour coding system that sums horizontal and vertical components in a display independently and evaluates the sums in a central "recognizor" (Dodwell, 1964), but they can also be interpreted in terms of what we know of the contour coding properties of retinal receptive field organization in the mammalian visual system. Hubel \& Wiesel $(1962,1968)$ have found neurons in the visual cortex of cats and monkeys that fire selectively for contours or edges in particular orientations (and positions, in the case of their "simple" fields). These units not only detect contour orientation, but also detect contour extent, in the sense that a unit's output increases as the length of contour in the preferred orientation is increased, up to a limit set by the size of its receptive field on the retina.
Consider now the population of cortical units that fire selectively for horizontal contours (Set $\mathrm{H}$ ) and those that fire selectively for vertical contours (Set V). Some members of each set will be activated by the "row" figure, but the $\mathrm{H}$ units will tend to have a stronger output. The reason for this is that the small squares in each row are closely spaced and will tend to produce summation of excitation in the horizontal fields. Summation of excitation in vertical receptive fields for the "row" pattern, on the other hand, will be much less, since contours are more widely separated in the vertical dimension. In fact, if the summation is linear, the output for $\mathrm{H}$ units should be about four times as great as for $V$ units, since the spacing is in the ratio 4:1. An exactly analogous argument applies to the "column" figure; for it, V units should give a higher output than should $H$ units. Thus, the original discrimination between "rows" and "columns" can plausibly be attributed to differential rates of firing for $\mathrm{H}$ and $\mathrm{V}$ units. For "rows," $\mathrm{H}$ output is higher than is V output, and for "columns," it is the reverse. It is clear that the smooth stripes will also affect $\mathrm{H}$ and $\mathrm{V}$ units differentially in the same sort of way, but the differences between $\mathrm{H}$ and $\mathrm{V}$ outputs for each shape will be even stronger than for the "row" and "column" patterns. In fact, we can show that the relative rates of firing give a very neat explanation of the anomalous transfer effects. Suppose $\mathrm{S}$ has been trained with "rows" positive, then the discrimination it has learned is: $\hat{H}>\hat{V}$ is positive and $\dot{V}>\hat{H}$ is negative, where $\hat{H}$ and $\hat{V}$ are the average outputs for $\mathrm{H}$ and $\mathrm{V}$, respectively. The smooth horizontal stripes are now shown with the "rows." Since there is more $H$ summation and less $V$ summation for stripes, $\mathrm{H}>\mathrm{V}$ now implies that horizontal stripes are the "positive" shape; although both patterns give $\hat{H}>\hat{V}$, the difference is greater for stripes (the "positive" signal is stronger or more redundant for the stripes). There is almost no $\mathrm{V}$ output for horizontal stripes, but there is some for "rows"; since the "rows" pattern has the bigger $\hat{V}$, it will tend to be treated as negative. The negative anomalous transfer can be treated in the same way. Here, $\hat{\mathrm{V}}>\hat{\mathrm{H}}$ implies negative, and vertical stripes give both a bigger output on $\hat{v}$ and a bigger difference between $\hat{V}$ and $\hat{H}$ than does the "column" pattern. When the two are shown together, the "column" pattern is preferred because the vertical stripes are "more negative."

Our paradigm is symmetrical with respect to horizontality-verticality, receptive-field properties, and positive-negative training conditions, so that a precisely analogous argument applies where "rows" are negative, "columns" positive, in initial training. Thu fact that neither monkey showed initial preference for smooth stripes in the same orientation as the "rows" or "columns" (nor preference for either of these over stripes) shows that the transfer results are genuine effects of the discrimination training. Also, "novelty" of the stripes can be ruled out, since an explanation in terms of a tendency to choose novel shapes would predict the opposite of what happened on negative transfer trials (see also Dodwell, in press).

The anomalous transfer results are not compatible with any traditional "continuity" type of explanation of discrimination learning in which positive and negative "cues" are summed in the two patterns (Spence, 1936) or are "sampled" from trial to trial, as is proposed in stochastic learning models (Restle, 1955; Atkinson \& Estes, 1963). On the other hand, the interpretation in terms of analysis by contour-detection units shows that the series horizontal stripes-"rows"-"columns"-vertical stripes forms a set of points on a single "horizontality-verticality" continuum, so that the anomalous transfers can be interpreted as cases of transposition behavior after all, in accordance with the classical analysis of Spence (1936). But the most important point to make is that the results can only be understood by taking account of the contour-coding system of the organism, which "structures" the stimulus input in a particular way.

We are puzzled by the fact that negative anomalous transfer only showed up after the positive anomaly had been elicited: No good explanation is readily apparent, but we hope to investigate the phenomenon experimentally in greater detail. The fact that anomalous transfers have been found in three species of mammal, using different apparatus and training techniques, suggests that this is not an isolated curiosity but a genuine function of the mammalian contour coding system. Yet it can scarcely be universal; one would not expect to get such transfer results with human adults. Transposition behavior is known to be influenced by the ability to attach verbal labels to discriminanda, however, so it would be interesting to know if the effects can be demonstrated in human infants.

\section{REFERENCES}

ATKINSON, R. C., \& ESTES, W. K. Stimulus sampling theory. In R. D. Luce, R. R. Bush, and E. Galanter (Eds.), Handbook of mathematical psychology. New York: Wiley, 1963. Pp. 121-269.

DODWELL, P. C. A coupled system for coding and learning in shape discrimination. Psychological Review, 1964, 71, 148-159.

DODWELL, P. C. Anomalous transfer effects affer shape discrimination training in the rat. Psychonomic Science, 1965, 3, 97-98.

DODWELL, P. C. Anomalous transfer effects after pattern discrimination training in rats and 
squirrels. Journal of Comparative and Physio logical Psychology (in press).

HUBEL, D. G.. \& WESEL, T. N. Receptive fields, binocular interaction and functional architecture in the cat's visual cortex. Joumal of Physiology, 1962, 160, 106-154.

HUBEL, D. H., \& WIESEL, T. N. Receptive fields and functional architecture of monkey striate cortex. Joumal of Physiology, 1968, 195, 215-243.

KRECHEVSKY, I. An experimental investigation of the principle of proximity in the visua perception of the rat. Journal of Experimental Psychology, 1938, 22, 497-523.
RESTLI, F. A theory of discrimination learning. Psychological Review, 1955, 62, 11-19.

SPENCE, $K$. W. The nature of discramination learning in animals. Psychological Review. $1936,43,427-449$.

THOMPSON, T., SEAL, D., \& BLOOM, W, A suspended platform for use in chronic restraint of monkeys. Journal of the Experimental Analysis of Behavior, 1966, 9, 146.

\section{NOTE}

1. This research was supported by Grant APA 44 from the National Research Council of Canada to the first author, which is gratefully acknowledged.

\section{Amnesic effects of cooling and heating in mice ${ }^{1}$}

\author{
JONATHAN KANE and MURRAY E. \\ JARVIK, Albert Einstein College of \\ Medicine, 1300 Morris Park Ave., Bronx, \\ N.Y. 10461
}

Albino CF1 female mice were heated with a diathermy unit immediately following training on a single-trial passive-avoidance task. Retention, tested $24 \mathrm{~h}$ later, was significantly impaired only in those animals that had heat-induced convulsions. Even elevations of temperature more than $5 \operatorname{deg} C$ above normal, whenever they did not produce convulsions, failed to produce amnesia. These results indicate that even the strong stimulation provided by hyperthermia is not sufficient in itself to produce amnesia.

A wide variety of posttrial treatments have been shown to influence memory in mice and rats (Jarvik, 1968; McGaugh, 1968). There are conflicting reports in the literature on the ability of cooling or heating to impair memory. Some investigators have failed to find any effect of cooling on either learning or memory in rats or hamsters (Andjus et al, 1956, in rats; Mrosovsky, 1963, in rats; Ransmeier \& Gerard, 1954, in hamsters). Others have reported impairment of memory in mice by cooling or heating (Beitel \& Porter, 1968; Jacobs \& Sorenson, 1969; Sudak \& Essman, 1962). Cerf \& Otis (1957) have reported that heating goldfish after a learning trial impaired their memory, and Agranoff (1967) reported that cooling goldfish prolonged the consolidation interval. Since our version of the one-trial passive-avoidance test (Jarvik \& Kopp, 1967) is sensitive to a variety of amnesic treatments [e.g., electroconvulsive shock (ECS), cycloheximide, and brain puncture], we thought it would be instructive to compare heating and cooling with these treatments in this particular test.

\section{SUBJECTS}

Female albino mice, CF1, obtained from Carworth Farms and weighing 18-20 g, were used. They were approximately 8-9 weeks of age at the time of the experiment and were housed in groups of eight in metal cages with free access to food pellets and water.

\section{APPARATUS AND PROCEDURE}

A trough-shaped two-compartment step-through apparatus was used (Jarvik \& Kopp, 1967). The mouse was placed in the outer, illuminated, smaller compartment facing a small opening to the larger, dark chamber, thereby activating a timer. When the mouse stepped into the larger, dark compartment and reached the third pair of metal plates that served as a floor, the timer stopped and a shock was delivered ( $800 \mathrm{~V} \mathrm{ac}$ through 2 megohms in series, causing about 320 microamperes root mean square $(\mathrm{rms}) \pm 20 \%$ flow for $0.8 \mathrm{sec}$ ). The animal usually received one and no more than three such shocks before running out into the smaller, lit compartment. This one-trial learning procedure generally took less than $20 \mathrm{sec}$.

Immediately after receiving this treatment, the mouse was removed from the step-through apparatus, and its rectal temperature was taken by means of a digital thermocouple thermometer. For heating, the animal was then placed on a table under an overturned glass jar. A diathermy unit from a Raytheon Microtherm machine, Model CMD5, was placed directly over the jar (Millichap, 1960). Treatments varied in time of exposure (90 to $180 \mathrm{sec}$ ) and in percentage of total power put out by the machine (30\% $\pm 5 \%$ in the treatment group and $0 \%$ in the control group). The step-through latencies were redetermined $24 \mathrm{~h}$ later with an upper limit of $300 \mathrm{sec}$.

\section{RESULTS}

Six groups of animals were tested. Groups A to $C$ were preliminary experiments to determine if raising of body temperature to subconvulsive temperatures caused memory deficits. It also served to establish the approximate length of exposure to the diathermy apparatus that was needed to cause convulsions in some of the animals and not kill them, while, at the same time, allowing other animals to be heated to temperatures below the convulsive threshold.

Control animals are Ss that were not heated. However, in Groups D and E, in which some heated Ss convulsed, the heated Ss that failed to do so serve as a control group. They, in turn, are compared with the nonheated control group in determining the effect of subconvulsing temperature elevations on memory. Animals that died as a result of the heat treatment and thus could not be retested after $24 \mathrm{~h}$ ( 2 in Group C, 10 in Group E, and 6 in Group F) are not included in Table 1.

Inspection of the results summarized in Table 1 shows that animals that were heated but failed to convulse did not show a significant memory deficit. Those animals exhibiting tonic-clonic convulsions (labeled "yes" in Table 1) showed retrograde amnesia for the one-trial learning experience $(p<0.01)$ when compared with those labeled "none." When retested after $24 \mathrm{~h}$, their latencies for entrance into the dark chamber were not significantly different from naive Ss during their first-trial learning experience. There was no significant difference between the nonconvulsing heated animals and the nonheated animals, none of whom, of course, convulsed.

The exact temperature at which convulsions occurred was difficult to determine since the rectal temperatures obtained in surviving Ss were lower than core temperatures taken on Ss that died in convulsions. This may account for the fact that one group without convulsions (Group D, 30\% power) reached a median temperature of $43.6 \mathrm{deg} \mathrm{C}$, while another group (Group C, 30\% power), with convulsions, reached a median temperature of $42.5 \mathrm{deg}$ C.

\section{DISCUSSION}

These experiments demonstrate that extreme changes in body temperature perse do not impair memory in our one-trial learning situation. Only when the temperature of the animals was raised sufficiently to produce a convulsion was retrograde amnesia produced. 